\title{
Determination of the miscibility gap in the solid solutions series of methylammonium lead iodide/bromide
}

\author{
Alexandra Franz ${ }^{\text {a*, Daniel M. Többens }}{ }^{\text {a }}$, Julia Steckhan ${ }^{\text {ab }}$ and Susan Schorr ${ }^{\text {ac }}$ \\ ${ }^{a}$ Structure and Dynamics of Energy Materials, Helmholtz-Zentrum Berlin für Materialien und Energie, \\ Hahn-Meitner-Platz 1, Berlin, 14109, Germany \\ bInstitute of Chemistry, University of Potsdam, Karl-Liebknecht-Str. 24-25, Potsdam, 14476, Germany \\ 'Institute of Geological Sciences, Freie Universität Berlin, Malteserstr. 74-100, Berlin, 12249, \\ Germany
}

Correspondence email: alexandra.franz@helmholtz-berlin.de

Synopsis We present the determination of the miscibility gap and of the maximum degree of $\mathrm{Cl}$ substitution in the hybrid perovskite solid solution series $\mathrm{CH}_{3} \mathrm{NH}_{3} \mathrm{~Pb}(\mathrm{I}, \mathrm{Cl})_{3}$, relevant for photovoltaic applications, based on high-resolution synchrotron powder X-ray data.

\section{Abstract}

Perovskites are widely known for their enormous possibility of elemental substitution, which leads to a large variety of physical properties. Hybrid perovskites such as $\mathrm{CH}_{3} \mathrm{NH}_{3} \mathrm{PbI}_{3}\left(\mathrm{MAPbI}_{3}\right)$ and $\mathrm{CH}_{3} \mathrm{NH}_{3} \mathrm{PbCl}_{3}\left(\mathrm{MAPbCl}_{3}\right)$ are perovskites with an $\mathrm{ABX}_{3}$-structure, where $\mathrm{A}$ is an organic molecule, $\mathrm{B}$ a lead (II)-cation and $\mathrm{X}$ a halide anion such as iodine (I) or chlorine (Cl). While $\mathrm{MAPbCl}_{3} \mathrm{crystallizes}$ in the cubic space group $P m \overline{3} m, \mathrm{MAPbI}_{3}$ belongs to the tetragonal space group $14 / \mathrm{mcm}$. The substitution of I by $\mathrm{Cl}$ leads to an increased tolerance against humidity but is challenging or even impossible due to their large difference in ionic radii. Here, we examine systematically the influence of an increasing $\mathrm{Cl}$ content in the reaction solution. Powders were synthesized by two different routes depending on the I:Cl ratio. High-resolution synchrotron $\mathrm{X}$-ray data are used to establish values for the limits of the miscibility gap which are 3.1(1.1) mol-\% $\mathrm{MAPbCl}_{3}$ in MAPI and 1.0(1) mol-\% $\mathrm{MAPbI}_{3}$ in $\mathrm{MAPCl}^{\mathrm{T}} \mathrm{The}$ establishment of relations between average pseudo-cubic lattice parameters for both phases allows a determination of the degree of substitution from the observed lattice parameters. 


\section{Introduction}

When Gustav Rose discovered a mineral which he named after the Russian mineralogist Lev A. Perovskij back in 1839, he for sure never thought that his discovery would have such a huge impact on today's photovoltaic research. Synthetic materials with $A_{B X}$ structure (for the sake of simplicity called "perovskites"), show - due to their chemical and structural variability - a wide bandwidth of physical properties. Besides "classic" oxide perovskites a group of organic-inorganic ("hybrid") halide perovskites came in forefront of research. Instead of A being a single cation a whole organic molecule is present in the structure (e.g. methylammonium, abbreviated in the following as MA).

$\mathrm{MAPbl}_{3}$ exhibits excellent solar conversion efficiencies of $22.1 \%$ (NREL, 2017) but is affected by humidity. $\mathrm{MAPbCl}_{3}$ on the contrary is stable in air. Doping $\mathrm{MAPbl}_{3}$ with $\mathrm{Cl}$-ions improves the air stability and furthermore, leads to an increased charge transfer, a lower crystal impedance and a lowered recombination rate (Unger et al., 2014; Ng et al., 2015; Yin et al., 2015). Moreover, it was proven that the chlorine impacts positively the growth and morphology of hybrid perovskite thin films (Yu et al. 2014; Yantara et al., 2015)

The question in dispute is, how much chlorine can be incorporated? In this regard several contradictory results were published. Unger et al. (2014) stated a chlorine content up to $10 \%$, revealed via X-ray fluorescence analysis, Colella et al. (2013) described the $\mathrm{Cl}$ incorporation as below 3 - 4 \% (powder Xray diffraction). Starr et al. (2015) and Cojocaru et al. (2015) published an inhomogeneous distribution of chlorine in thin films with Starr et al. (2015) reporting surface depletion and interface enrichment. Grätzel (2014) referred to a second-hand report of $\mathrm{MAPbl}_{3}$ formed by reaction of $\mathrm{CH}_{3} \mathrm{NH}_{3}$ l solution with $\mathrm{PbCl}_{2}$ not containing any chlorine at all according to lattice parameters.

Aim of our investigation is the determination of the miscibility and the establishment of a quantitative relation between degree of substitution and lattice parameters.

\section{Synthesis}

The synthesis of the solid-solution series $\operatorname{MAPbX}_{3}(\mathrm{X}=\mathrm{I}, \mathrm{Cl})$ was done by two different routes for the iodine-rich and the chlorine-rich samples, respectively. It is not possible to use the same procedure for all samples, due to the poor solubility of methyl ammonium chloride.

$\mathrm{MAPbl}_{3}$ and the iodine-rich solid solution powder samples were synthesised using the method after Baikie et al. (2013) and Colella et al. (2013). Synthesis was done within a glove box under inert gas atmosphere in order to avoid a contamination of the sample, in particular by moisture or oxygen (Green et al., 2014; Grätzel (2014). For the pure $\mathrm{MAPbl}_{3}$ 2.230g lead iodide ( $\mathrm{Pbl}_{2}$, Sigma-Aldrich, 99\%) and $0.769 \mathrm{~g}$ methyl ammonium iodide (MAl, Sigma-Aldrich, $98 \%$ ) were dissolved in a $25 \mathrm{ml}$ double neck 
bottom flask in $4 \mathrm{ml}$ gamma-butyrolactone (GBL, $\leq 99 \%$ for synthesis, Roth) and homogenised for $20 \mathrm{~h}$ at $60{ }^{\circ} \mathrm{C}$ in a heat-on block system. After cooling down, a yellow solution resulted. The solvent was subsequently evaporated by heating $\left(T \leq 110^{\circ} \mathrm{C}\right)$ the solution in a petri dish, resulting in the formation of dark grey crystalline $\mathrm{MAPbl}_{3}$ powder.

The first step of iodine rich solid solution series synthesis was weighing out stoichiometric amounts of $\mathrm{Pbl}_{2}, \mathrm{MAl}$ and methylammonium chloride (MACl, Sigma-Aldrich, for synthesis) into a $25 \mathrm{ml}$ double neck bottom flask and dissolving it in the equivalent amount of GBL. N,N-dimethyl formamide (DMF, 99,8\%, p.a., ACS, ISO, Roth) was used as supplementary solvent with increasing amount of MACl. Increasing the total volume of the reaction mixture prevents precipitation of $\mathrm{PbCl}_{2}$ during homogenisation procedure. Moreover, with increasing $\mathrm{Cl}$-content the initial homogenisation temperature was gradually raised up from $60^{\circ} \mathrm{C}$ to $85^{\circ} \mathrm{C}$.

This synthesis route turned out to be only sufficient up to a maximum weighed out chlorine content of 33\%. To achieve a higher $\mathrm{Cl}$-content lead(II) chloride ( $\mathrm{PbCl}_{2}, 99,999 \%$, Sigma-Aldrich) had to be added additionally.

To avoid the above outlined solubility problems the preparation of $\mathrm{MAPbCl}_{3}$ and the chlorine rich solid solution powder samples was done following the procedure after Leguy et al. (2015). Protective atmosphere in this case was ensured by carrying out the whole procedure under $\mathrm{N}_{2}$ inert gas in the fume hood. All educts were weighed in stoichiometric ratio.

A methylamine solution ( $40 \mathrm{wt} \%$ in $\mathrm{H}_{2} \mathrm{O}$, Sigma-Aldrich) in a $100 \mathrm{ml}$ triple neck round bottom flask with dropping funnel was cooled down to $5^{\circ} \mathrm{C}$ and neutralised with concentrated aqueous hydrochloric acid $\left(\mathrm{HCl}\right.$, fuming, 37\%, p.a., Roth). To prevent the formation of $\mathrm{PbCl}_{2}$ as a chemical by-product, excess $\mathrm{HCl}$ (approx. $2 \mathrm{ml}$ ) was additionally added. This solution was heated in an oil bath to $100{ }^{\circ} \mathrm{C}$. An aqueous solution of $2.21 \mathrm{~g}$ lead-(II) acetate trihydrate $\left(\mathrm{Pb}\left(\mathrm{CH}_{3} \mathrm{COO}\right)_{2} 3 \mathrm{H}_{2} \mathrm{O}\right)$, 99.5\%, p.a., Roth) and $9 \mathrm{ml} \mathrm{H}_{2} \mathrm{O}$ was then added dropwise. A fine white precipitate resulted. To avoid hydration of the product at temperatures below $40^{\circ} \mathrm{C}$, the reaction mixture was filtered at $80^{\circ} \mathrm{C}$, washed with ethanol (absolute $99.8 \%$, Sigma-Aldrich) and afterwards dried at $50^{\circ} \mathrm{C}$ for $1-2$ days in drying cabinet.

The preparation of $\operatorname{MAPb}(\mathrm{Cl}, \mathrm{I})_{3}$ solid solution followed the above described procedure but instead of pure $\mathrm{HCl}_{\mathrm{aq}}$ a 99:1 solvent mixture of concentrated hydrochloric and hydriodic acid $\left(\mathrm{HI}, 57\right.$ wt\% in $\mathrm{H}_{2} \mathrm{O}$, distilled, stabilized, 99.95\%, Sigma-Aldrich) was used.

\section{Experimental}

X-ray powder diffraction data were collected at the Diffraction station of KMC-2 beamline (BESSY II, HZB (Helmholtz Zentrum Berlin, 2016), using a radiation energy of $8048 \mathrm{eV}$ (equivalent to a wavelength 
of $1.5406(1) \AA ̊$ ). The sample was mounted at ambient conditions in symmetric reflection geometry on a rotating zero-scattering silicon single-crystal sample holder. As humidity inside the BESSY II experimental hall was very low, no degradation of the samples occurred during the measurements. A VANTEC 2000 area detector was used, allowing the accumulation of very high counting rates in short time; total measuring time for each diffraction pattern in the $2 \theta$-range $5-85^{\circ}$ was only 25 minutes. As a result of the low intrinsic detector background the detection limit for secondary phases, in particular the presence of a second perovskite phase, was extremely low. The zero point of the detector position has been determined directly before conducting data collection to an accuracy $<0.01^{\circ}$. The sample height was determined for each individual sample to an accuracy $<0.01 \mathrm{~mm}$, with the diameter of the detector circle being $560 \mathrm{~mm}$. As a result, no significant systematic peak shift was observed. To further increase the reliability of the $2 \theta$ positions, 20 wt\% LaB6 SRM 660b was added to the powder samples as an internal standard; the certified lattice parameter for a temperature of $22.5^{\circ} \mathrm{C}$ of 4.15689 (8) $\AA$ was used in analysis. This procedure results in a very high reliability of the lattice parameters refined from the data.

The calculation of the lattice parameters $a$ and $c$ was performed with a LeBail refinement using Fullprof (Rodriguez-Carvajal, 1993). Semi-quantitative phase analysis was done with the same software. The crystal structures of $\mathrm{MAPbl}_{3}$ (Franz et. al, 2016) and $\mathrm{MAPbCl}_{3}$ (Baikie et. al, 2015) used in this were taken from literature.

\section{Results and discussion}

The formation of solid solutions is favoured if the difference of the ionic radii of the substitution partners is maximum 15\% and the crystal structures are isotypic (Borchard-Ott, 1997). Taking the ionic radii for iodine and chlorine from Shannon (1976), the difference is about $21 \%$. A rather low miscibility is thus expected.

In the following, the terms $\mathrm{MAPbl}_{3}$ and $\mathrm{MAPbCl}_{3}$ will be used for the pure end members (diffraction pattern 1a and 1d). The terms MAPI and MAPCl will be used for any compound within the solid solution series, whose composition is on the respective side of the miscibility gap and which thus has essentially the crystal structure of the pure end member, but may exhibit a degree of anion substitution. Where a quantitative degree of substitution $\mathrm{x}$ is given, this refers to the molar percentage of $\mathrm{Cl}$ among the total anions $(\mathrm{Cl}+\mathrm{I})$; note that this is equivalent to the molar percentage of $\mathrm{MAPbCl}_{3}$.

The synchrotron experiment data clearly show single phased solid solution powders for all samples with of less than $3.0 \mathrm{~mol} \% \mathrm{Cl}$ among the total anion content. (fig1b and fig S1). Above this value a phase separation takes place, two phases are formed (see fig.1, c and S1, fig S2, S3 and S4). This second phase 
can be identified from the powder diffraction pattern as MAPCl. Increasing the $\mathrm{Cl}$ content up to 36.7 mol\% results in qualitatively the same phase composition, with increasing fraction of the MAPCI phase.

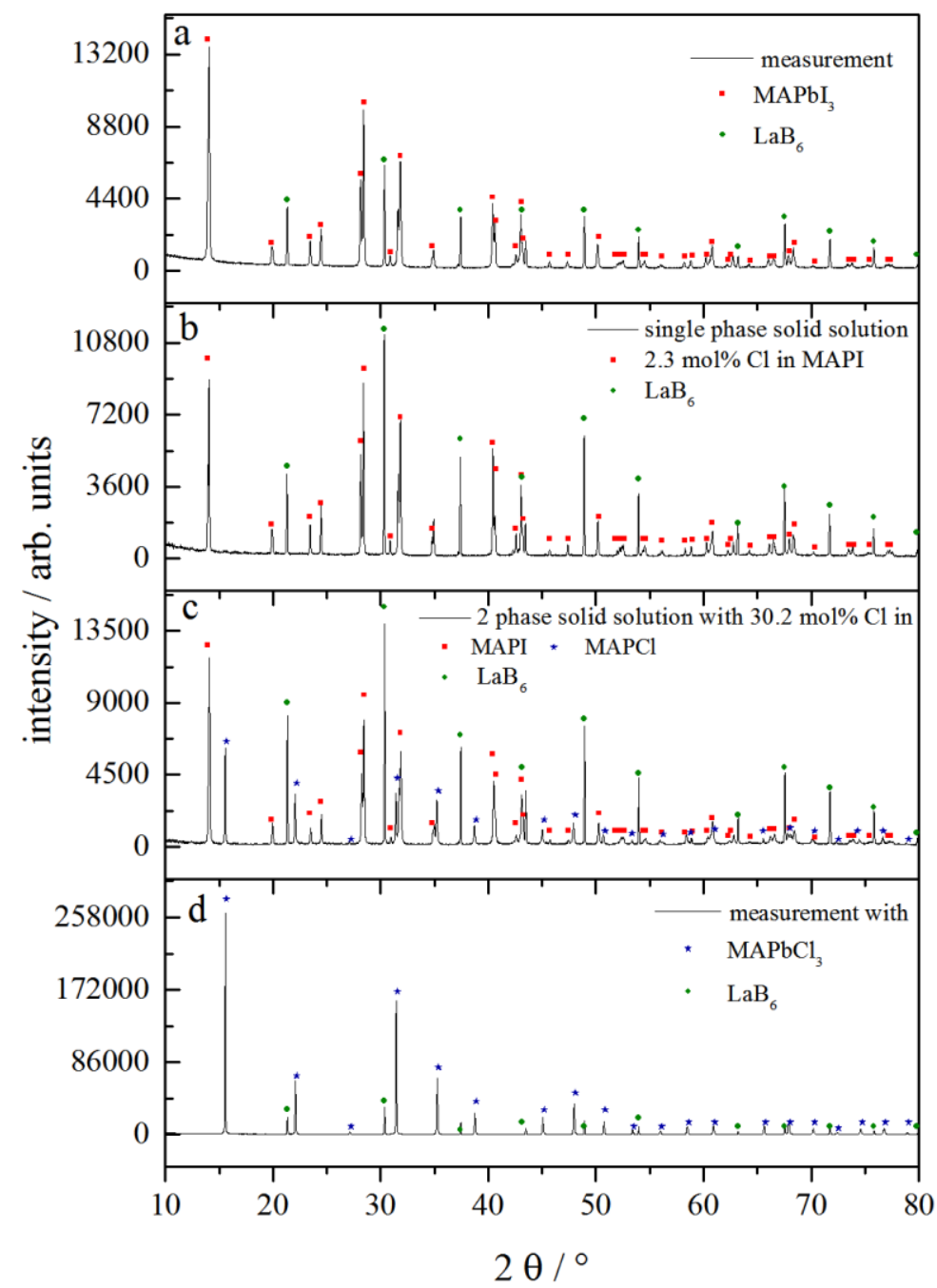

Figure 1 Synchrotron X-ray diffractograms of the solid solution end members $\mathrm{MAPbI}_{3}(\mathrm{a}), \mathrm{MAPbCl}_{3}$ (d), the iodine rich single phase solid solution with $2.3 \% \mathrm{Cl}$ (b), 2 phase solid solution with $30.2 \mathrm{~mol} \%$ $\mathrm{Cl}(\mathrm{c})$.

From the first appearance of $\mathrm{MAPCl}$ the iodine-rich edge of the miscibility gap could already be established as $2.65 \pm 0.35 \mathrm{~mol}-\% \mathrm{Cl} /(\mathrm{Cl}+\mathrm{I})$. Semi quantitative phase analysis using the Rietveld method determined the amount of $\mathrm{MAPCl}$ phase as only $0.3(1)$ wt-\%, equivalent to $0.5(1) \mathrm{mol}-\%$, for the sample with totally $3.0 \mathrm{~mol}-\% \mathrm{Cl}$. These values refer to the composition of the sample only; the amount of $\mathrm{LaB}_{6}$ added for the diffraction analysis has been corrected. As the synthesis procedure used for the iodinerich samples is such that all anions remain in the product it is clear from this that some chlorine ions have to be incorporated into the crystal structure of the MAPI phase. A direct subtraction of the $0.5(1)$ mol-\% MAPCI phase indicates a content of $2.5(1)$ mol-\% of $\mathrm{Cl}$ in the MAPI phase at the edge of the 
miscibility gap. However, at this point of the analysis the possibility of an undetected amorphous phase containing some of the chlorine could not be excluded, as such a small fraction of amorphous material would be all but undetectable by diffraction methods.

A better quantitative understanding of the uptake of chlorine into the structure of MAPI and the corresponding miscibility gap can be derived from the composition dependence of the lattice parameters of the unit cell (table 1). The simplest approach is linear interpolation between the lattice parameters of the pure phases, $\mathrm{MAPbl}_{3}$ and $\mathrm{MAPbCl}_{3}$. Linearity of the lattice parameter of a solid solution is known as Vegard's law (Vegard, 1921 and 1928).

$\mathrm{MAPCl}$ at ambient conditions crystallizes in the cubic aristotype structure, space group $\operatorname{Pm} \overline{3} m, a \approx 5.7$ $\AA$. MAPI, on the other hand, at ambient conditions forms a superstructure with space group $I 4 / \mathrm{mcm}$ and lattice parameters of $a \approx 8.8 \AA, c \approx 12.6 \AA$, according to a $\sqrt{2} \times \sqrt{2} \times 2$ supercell. In order to compare the evolution of lattice parameters over the whole composition range, for MAPI the geometric mean pseudo-cubic lattice parameter $\langle a\rangle=\sqrt[3]{a^{2} c / 4}=\sqrt[3]{V / 4}$ has to be used. Taking the individual lattice parameters $a$ or $c$, scaled to the corresponding pseudo-cubic lattice, would give inconsistent estimations of the chemical compositions due to anisotropic expansion of the unit cell.

Assumption of Vegard's law allows assigning a degree of substitution $x$ from the lattice parameters of the pure phases using the following equation:

$$
x / \%=100 \cdot \frac{\langle a\rangle-\langle a\rangle_{M_{A P I_{3}}}}{\langle a\rangle_{\mathrm{MAPbCl}_{3}}-\langle a\rangle_{\mathrm{MAPbI}_{3}}}
$$

Pure $\mathrm{MAPbl}_{3}$ 's mean pseudo-cubic lattice parameter is $\left\langle\mathrm{a}>_{\mathrm{MAPb} 13}=6.2939(2) \AA\right.$, for pure $\mathrm{MAPbCl}_{3}$ it is $<\mathrm{a}>_{\mathrm{MAPBC} 13}=5.6850(2) \AA$. From this it is possible to calculate the substitution $\mathrm{x}$ for all phases. Plotting the lattice parameters against these substitution values (figure 2) of course gives an ideal linear plot, but provides an intuitive insight into the borders of the miscibility ranges. In particular, the phase whose lattice parameter deviates the most from its respective pure form can be identified easily. From this the limits of the miscibility gap are estimated to be $x=2.2 \mathrm{~mol}-\%$ for the replacement of iodine by chlorine in MAPI and on the chlorine-rich side $x=98.7 \mathrm{~mol}-\%$, that is up to $1.3 \mathrm{~mol}-\%$ of chlorine can be replaced by iodine in the MAPCl structure.

However, while linear interpolation is often applied in this way, it is also well established that Vegard's rule is not strictly fulfilled in non-ideal systems (Jacob et. al, 2007). This is the case in particular for systems with a miscibility gap, as the existence of such a region of immiscibility is a clear indicator that the constituents cannot replace each other in the structure easily.

The application of a second approach is thus in order. 


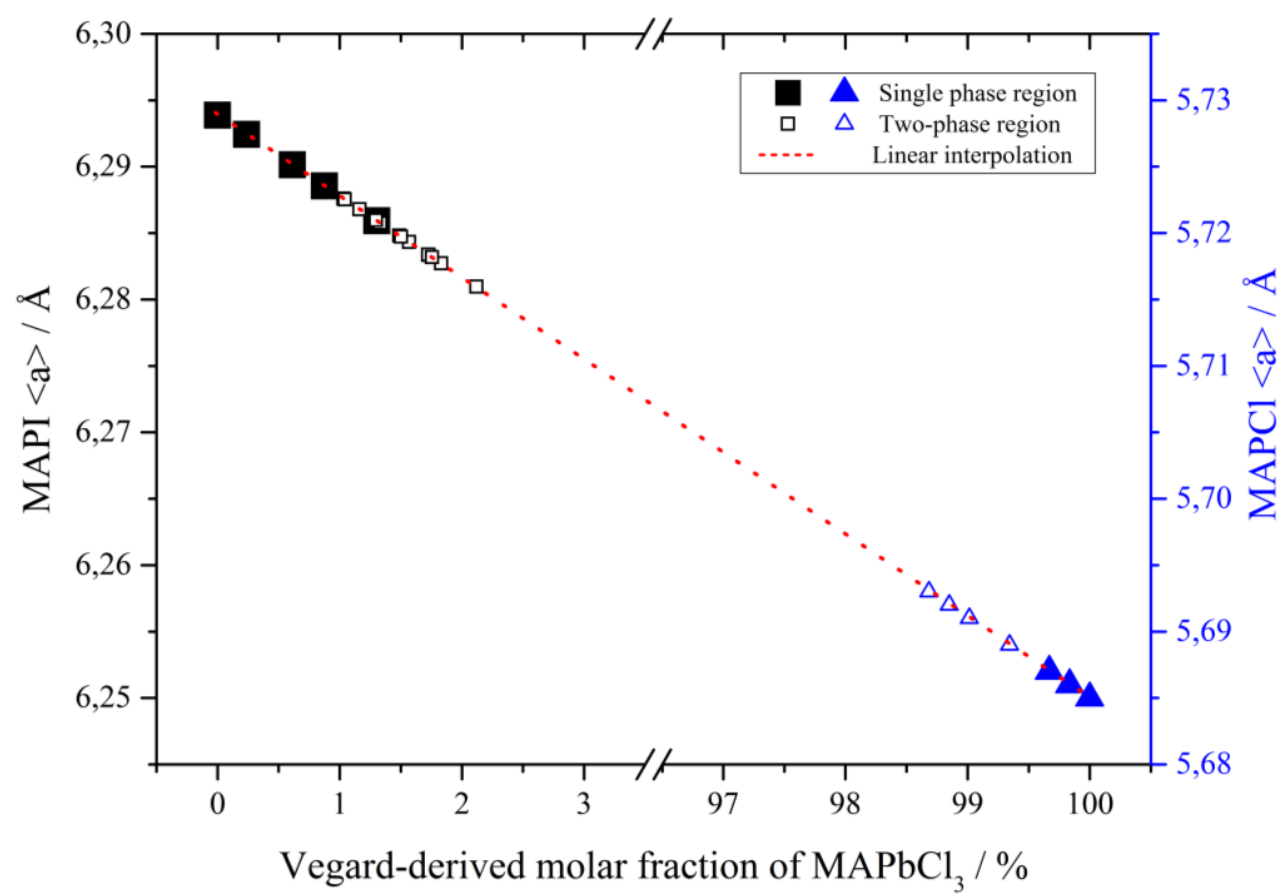

Figure 2 Visualization of the miscibility gap in $\operatorname{MAPb}\left(\mathrm{I},{ }_{x} \mathrm{Cl}\right)_{1-\mathrm{x}}$; the red dotted line indicates the change in the mean pseudo-cubic lattice parameter $<a>$ following Vegard's law. Estimated uncertainties from Rietveld refinement are $\pm 0.001 \AA$. The composition is expressed as molar fraction of $\mathrm{MAPbCl}_{3}$.

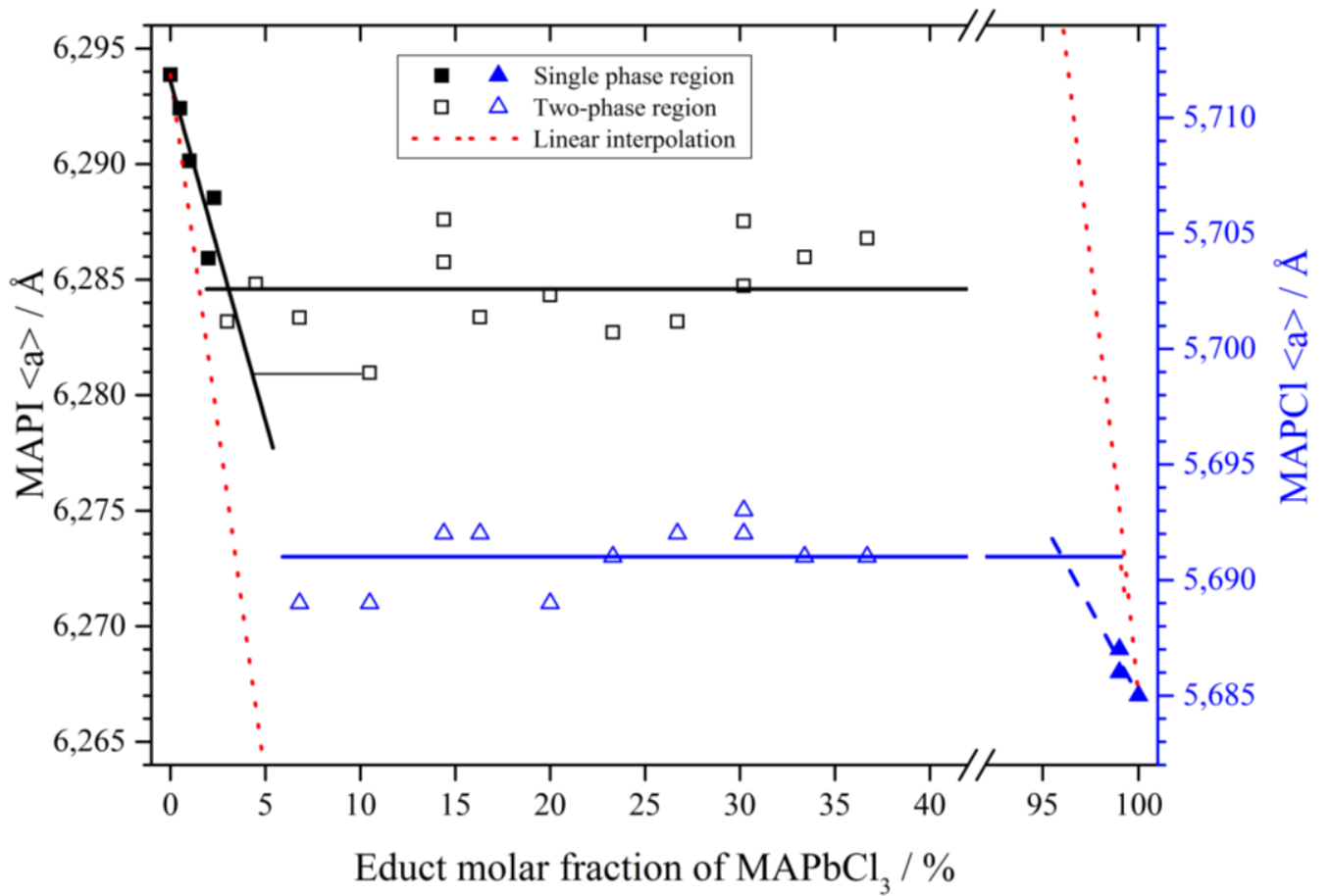

Figure 3 Geometric mean lattice parameter as function of molar fraction of $\mathrm{MAPCl}$ according to synthesis educts. Estimated uncertainties from Rietveld refinement are smaller than the symbols, but are 
obviously underestimated. Solid lines represent linear and constant fits of the respective data. The dotted lines represent expected ideal behaviour according to Vegard's law.

This second approach to define the miscibility gap makes use of the fact that during the synthesis there is no path for the heavy elements to escape and the amount of impurity phases is negligible. It can thus be assumed that the overall composition of the perovskite phases is the same as calculated from the educts.

In the iodine-rich single phase region, where only the MAPI perovskite phase is present, this composition directly corresponds to the composition of the perovskite phase. From this a linear dependency of the geometric mean lattice parameter can be derived (figure 3-filled black squares) as $<a>_{\text {MAPI }}=6.2936(11) \AA$ - 0.0029(8) $\AA / \mathrm{mol}-\% \mathrm{X}_{\text {MAPbC13. }}$.

An estimation of the maximum substitution of iodine by chlorine, the edge of the miscibility gap, can be derived by applying this relation to the lowest value of the average pseudocubic lattice parameter observed for any MAPI phase, which is 6.2810 (2) $\AA$. This would correspond to a substitution grade of $\mathrm{X}_{\mathrm{MAPBC} 33}=4.4(1.6) \mathrm{mol}-\%$ (see intersection in Fig. 3 - black thin line).

As the phase regions, in which mixing occurs, are narrow, uncertainties of the slope are necessarily high. However, the deviation from a simple linear interpolation between the pure phases is significant. Under ideal mixing conditions, the lattice parameters could be expected to follow such a linear interpolative behaviour as described by Vegard's law and calculated above. In this case the slope would be $-0.00609 \AA / \%$, about two times the observed value and significantly outside of the boundaries of uncertainty. This is not surprising. The lengths of the edges of the unit cell of MAPI might be defined by the size and tilting of the corner-connected $\mathrm{Pbl}_{6}$-octahedra making up the framework of the structure. However, the anions are interconnected by multiple hydrogen bonds formed with the methylammonium cation (Franz et. al, 2016). This stiffens the framework and stops it from full reacting to the substitution of small chlorine for larger iodine anions.

Within the two-phase region, neither of the both phases shows a significant slope in the lattice parameter. Average values are $<\mathrm{a}>_{\text {MAPl, } 2 \text { phase }}=6.2856(5) \AA$ and $<a>_{\text {MAPCl, } 2 \text { phase }}=5.6910(4) \AA$, respectively. This behaviour indicates a constant composition of the two phases within the two-phase region. This is in agreement with a strict miscibility gap covering the larger part of the composition range. The iodine-rich limit of this gap can be derived from the intersection of the two lattice parameter fits for MAPI as 3.1(1.1) mol-\%.

The various approaches place the miscibility edge between 2.2 and $4.4 \mathrm{~mol}-\% \mathrm{MAPbCl}_{3}$ in MAPI but, taking all parameters into account the value of 3.1(1.1) mol-\% is the most reliable one. The remaining comparatively high uncertainty is probably related to the observed scatter of the lattice parameters, 
which is much higher than expected from the estimated uncertainties of the refined lattice parameters (figure 3). This indicates that the exact value of the substitution limit depends on small details in the synthesis process.

For the chlorine-rich perovskites (figure 3, triangles) only a tentative estimation of the substitution limit can be derived. For the MAPCl forming as a second phase from iodine-rich educts (open triangles), their chemical composition cannot not be established with sufficient accuracy. Phase analysis with the Rietveld method is only semi-quantitative and retained an uncertainty of about 1 weight-\% for the fractions of the individual phases. This uncertainty is on the same scale as the expected substitution. On the other hand, for the single-phase MAPCl samples (closed triangles), the synthesis process does not preserve the composition of the educts, as $\mathrm{H}(\mathrm{Cl}, \mathrm{I})_{\text {aq }}$ has to be used in excess. It is not necessary for the degree of substitution of chlorine by iodine in the solid solution to be the same as the anion ratio in the acid (this assumption was used for the placement of these data points in figure 3 ). In fact, given the small range of miscibility, it is probable that the uptake of iodine in the solid solution is lower. However, this assumption delivers a lattice parameter dependency of $<a>$ MAPCl $=5.6850(7) \AA-0.0015(8)$ $\AA / \mathrm{mol}-\%$ ( $\left.\mathrm{X}_{\text {MAPbCI3 }}-100 \%\right)$.

The intersection with the average constant lattice parameter of MAPCI produced as secondary phase from iodine-rich educts gives than a maximum substitution of 4.0 mol-\% $\mathrm{MAPbl}_{3}$ into $\mathrm{MAPCl}$, albeit with a very high uncertainty. A more realistic assumption is that the lattice parameter in the chlorinerich part of the phase diagram follows Vegard's law. This is, because while small anions in place of large ones can fit loosely, the framework has to adapt in order to make space for large iodine anions replacing smaller chlorine ones. The intersection of the Vegard line with the average lattice parameter for the saturated MAPCl then gives a miscibility limit of only 1.0(1) mol-\% $\mathrm{MAPbl}_{3}$ in $\mathrm{MAPCl}$. However, perovskites synthesized with this $\mathrm{Cl} /(\mathrm{Cl}+\mathrm{l})$ ratio in the solution yielded only crystals with obviously lower substitution of only 0.2 and $0.3 \mathrm{~mol}-\%$.

\section{Summary}

This work establishes values for the limits of the miscibility gap in the solid solution system of $\mathrm{MAPb}(\mathrm{I}, \mathrm{Cl})_{3}$ perovskites with 3.1(1.1) $\mathrm{mol} \% \mathrm{MAPbCl}_{3}$ in MAPI and 1.0(1) $\mathrm{mol}-\% \mathrm{MAPbl}_{3}$ in $\mathrm{MAPCl}$. The establishment of relations between average pseudo-cubic lattice parameters for both MAPI and MAPCI phases allows a determination of the degree of substitution from the observed lattice parameters alone. 


\section{Acknowledgements}

The authors - especially J.S. - would like to acknowledge Prof. Andreas Taubert from University of Potsdam for his contribution to this paper.

\section{References}

Baikie, T., Barrow, N S., Fang, Y., Keenan, P. J., Slater, P. R., Piltz, R O., Gutmann, M., Mhaisalkar, S. G. \& White, T. J. (2015). J. Mater. Chem. A 3, 9298-9307.

Borchard-Ott, W. (1997). Kristallographie-eine Einführung für Naturwissenschaftler, $5^{\text {th }}$ ed., Springer Cojacaru,L. ; Uchida, S. ; Jena, A. K. ; Miyasaka, T. ; Nakazaki, J. ; Kubo, T. \& Segawa, H. (2015). Chem. Lett. 44, 1089-1091.

Colella, S., Mosconi, E., Fedel, P., Listorti, A., Gazza, F., Orlandi, F., Feroo, P., Besagni, T., Rizzo, A., Castelani, G., Gigli, G., De Angelis, F. \& Mosca, R. (2013). Chem. Mater. 25, 4613-4618. Franz, A., Többens, D. M. \& Schorr, S. (2016). Cryst. Res. \& Technology, 51(9), 534-540. Fullprof Suite, Rodriguez-Carvajal, J. (1993). Physica B, 192(1-2), 55-69.

Grätzel, M. (2014). Nat. Mater. 13, 838-842.

Green, M. A., Ho-Baillie, A. \& Snaith, H. J. (2014). Nat. Photon. 8, 506-514.

Helmholtz-Zentrum Berlin (2016). JLSRF 2, A49.

Jacob, K. T., Raj, S. \& Rannesh, L. (2007), J. Mater. Res. 98 (9), 776-779.

Leguy, A. M. A., Hu, Y., Campoy-Quiles, M., Alonso, M. I., Weber, O. J., Azarhoosh, P., Schilfgaarde, M. van, Weller, M. T., Bein, T., Nelson, J., Docampo, P. \& Barnes, P. R. F. (2015). Chem. Mater. 27, 3397-3407.

Ng, T.-W., Chan, C.-Y., LO, M.-F., Guan, Z. Q. \& Lee, C.-S (2015). J. Mater. Chem. A, 3, 90819085.

NREL (2017) https://www.nrel.gov/pv/assets/images/efficiency-chart.png Rose, G. (1839). De perowskite, fossili novo. in De novis quibusdam fossilibus quae in Montibus Uraliis inveniuntur. aG Schade, Berlin, 3-5.

Shannon, R. D. (1976). Acta Cryst. A 32, 751-767.

Starr, D. E., Sadoughi, G., Handick, E., Wilks, R. G., Alsmeier, J. H., Köhler, L., Gorgoi, M., Snaith, H. \& Bär, M. (2015). Energy Environ. Sci. 8, 1609-1615.

Unger, E. L., Bowring, A. R., Tassone, C. J., Pool, V. L., Gold-Parker, A., Cheacharoen, R., Stone, K. H., Hoke, E. T., Toney, M. F. \& Mcgehee, M. D. (2014). Chem. Mater. 26, 7158-7165.

Vegard, L. (1921). Z. Phys. 5, 17-26

Vegard, L. (1928). Z. Krist. 67 (1-6), 239-259.

Yantara, N., Yanan, F., Shi, C., Dewi, H. A., Boix, P. P., Mhaisalkar, S. G. \& Mathews, N. (2015). Chem. Mater. 27(7), 2309-2314.

Yin, W.-J., Yang, J.-H., Kang, J., Yan, Y. \& Wei, S.-H. (2015). J. Mater. Chem. A, 3, 8926-8942. Yu, H., Wang, F., XIE, F., Li, W., Chen, J. \& Zhao, N. (2014). Adv. Funct. Mater. 24, 7102-7108. 


\section{Supporting information}

The terms MAPI and MAPCI will be used for any compound within the solid solution series, whose composition is on the respective side of the miscibility gap and which thus has essentially the crystal structure of the pure end member, but may exhibit a degree of anion substitution.

\section{S1. Powder diffraction data with qualitative phase analysis}

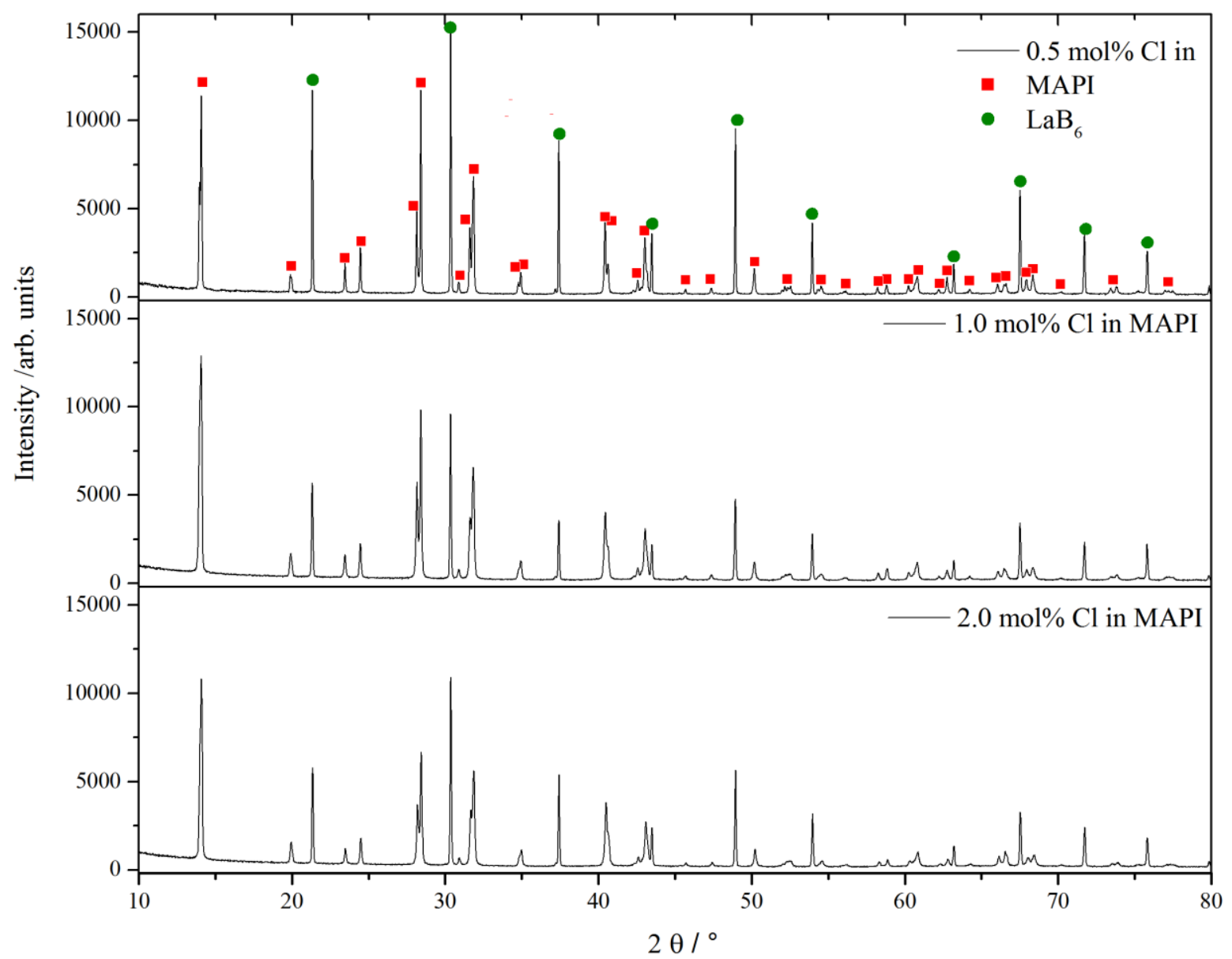

Figure S1 Diffractograms of the single phase samples of the iodine-rich side of the phase diagram. The red squares refer to the solid solution MAPI, green circles to $\mathrm{LaB}_{6}$ which was used as an internal standard to increase the accuracy of lattice parameters. 


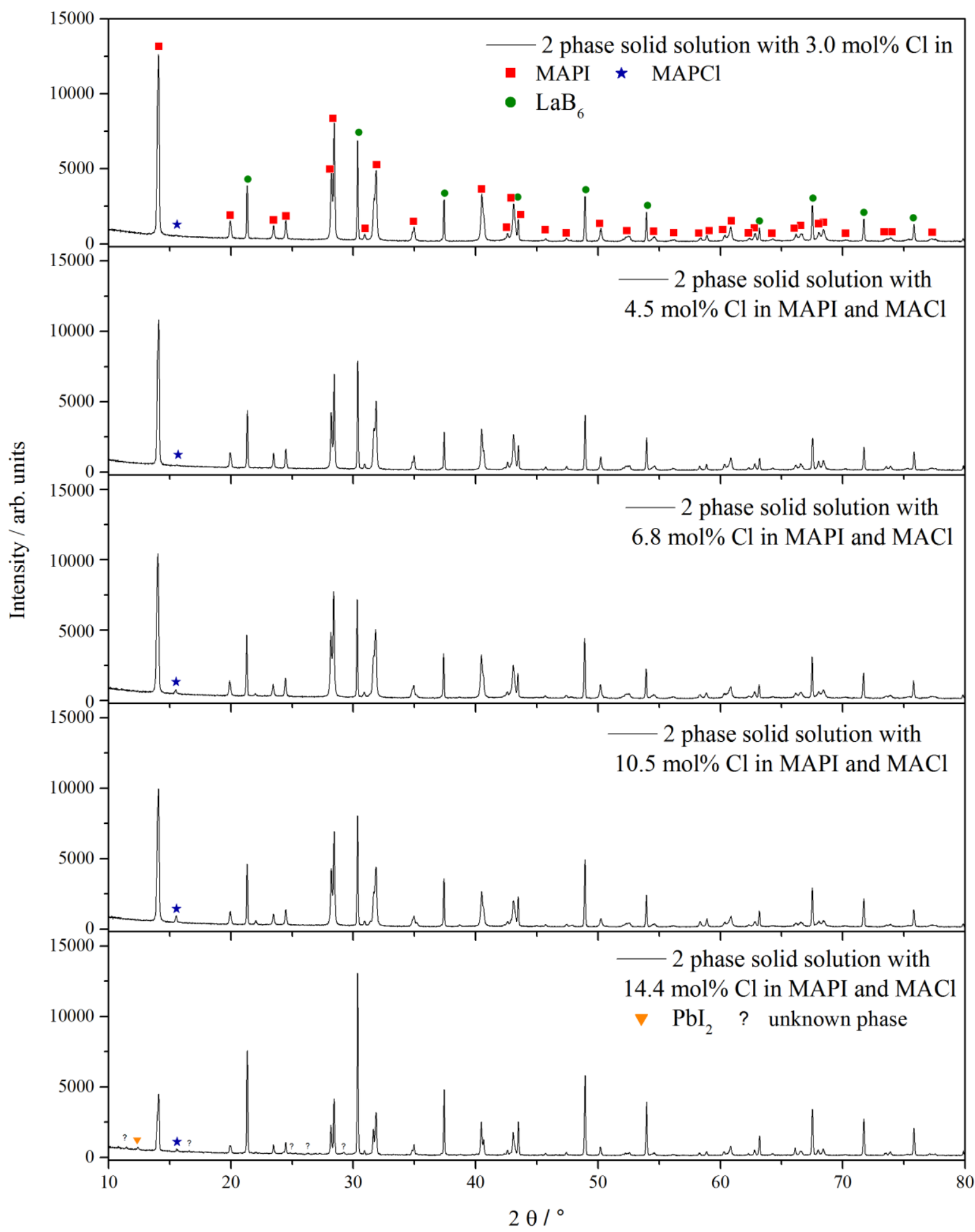

Figure S2 2 phase solid solutions with red squares referring to MAPI, blue stars referring to MACl, green circles to $\mathrm{LaB}_{6}$. The bottommost graphic show small impurities of $\mathrm{PbI}_{2}$. This shows the sensitivity of the synthesis method since exactly the same procedure was used for the sample with the same chemical composition show in Figure S3 (topmost graphic) 


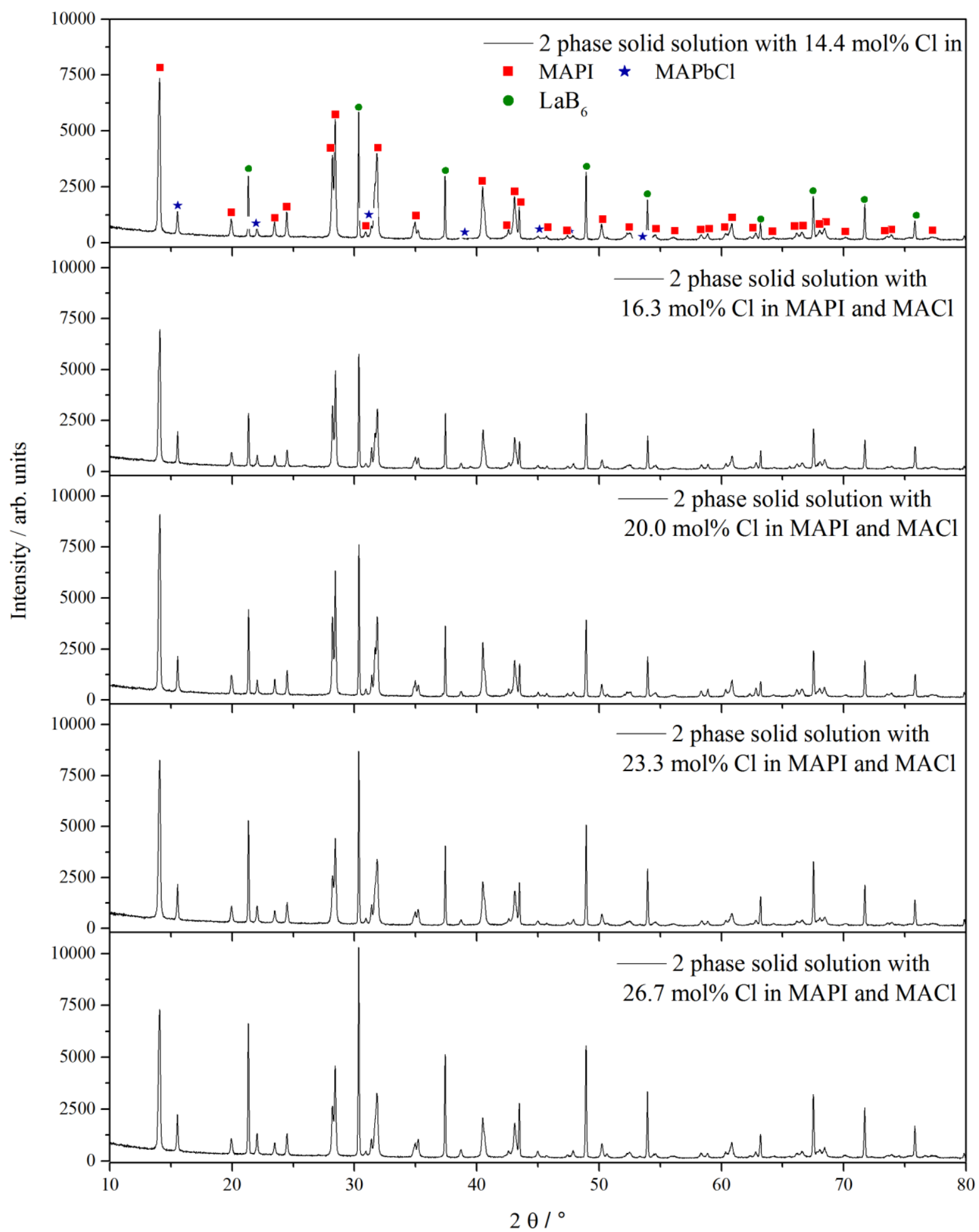

Figure S3 2 phase solid solutions with red squares referring to MAPI, blue stars referring to MACl, green circles to $\mathrm{LaB}_{6}$. 


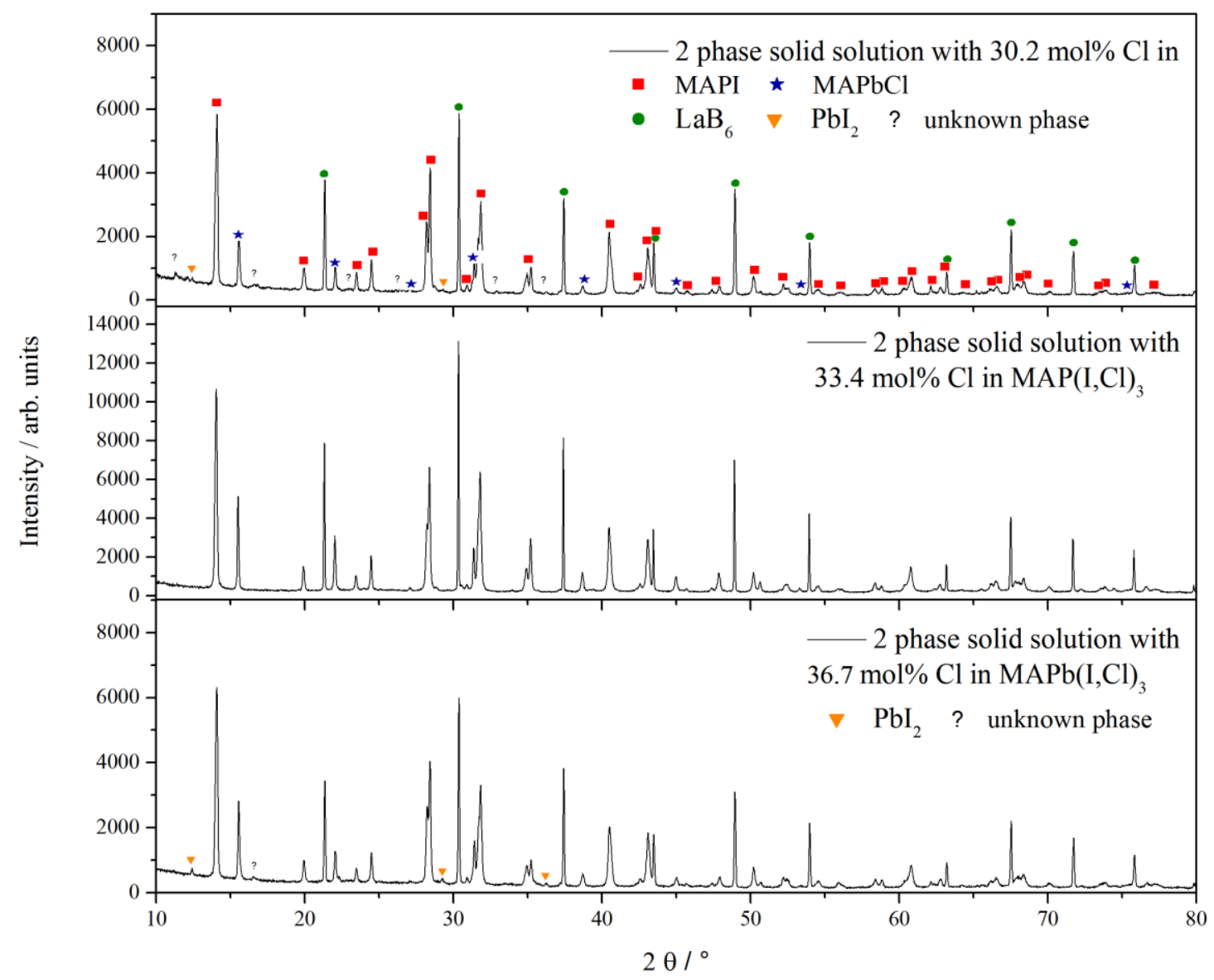

Figure S4 2 phase solid solutions with red squares referring to MAPI, blue stars referring to MACl, green circles to $\mathrm{LaB}_{6}$, yellow triangles to small impurities of $\mathrm{PbI}_{2}$.

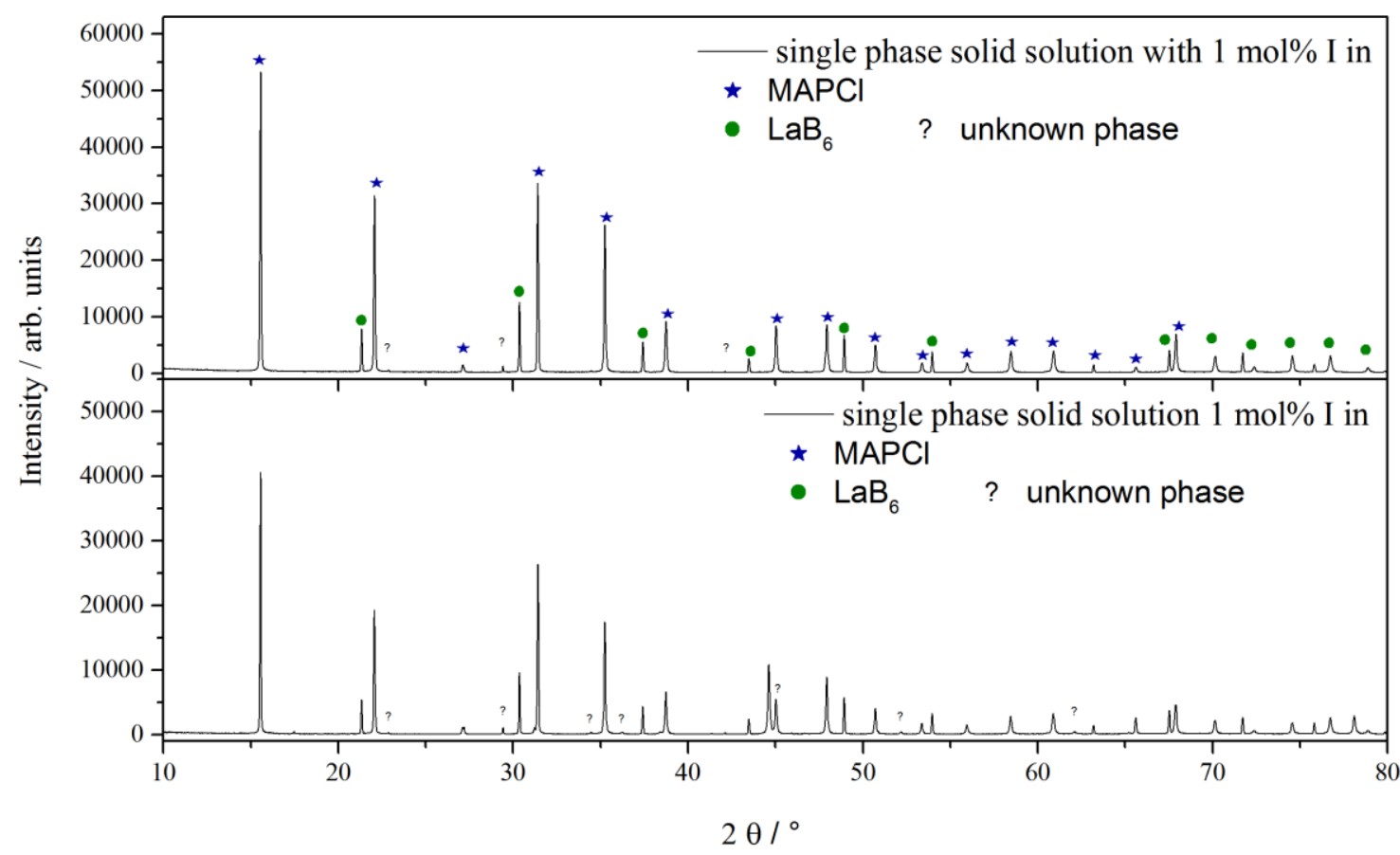


Figure S5 Single phase solid solution of $\mathrm{MAPCl}$ (blue stars) and $\mathrm{LaB}_{6}$ (green circles)

\section{S2. Lattice parameters of solid solution members}

Table S1 Phase formation and lattice parameters as function of the chemical composition. The $\mathrm{Cl}$ content is given as molar fraction $\mathrm{Cl} /(\mathrm{Cl}+\mathrm{I})$.

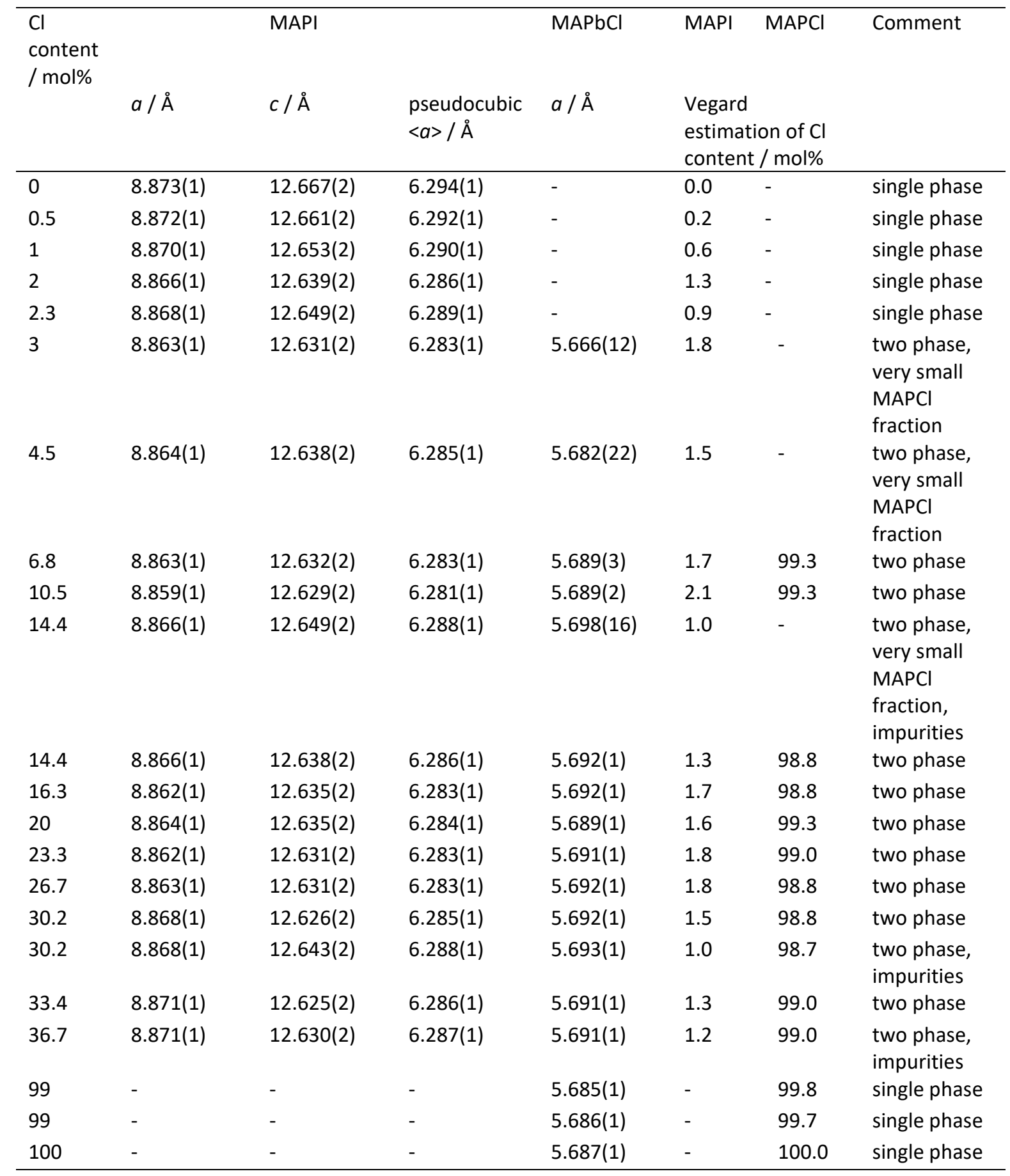

